\title{
A TWO-CARDINAL THEOREM AND A COMBINATORIAL THEOREM
}

\author{
SAHARON SHELAH ${ }^{1}$
}

\begin{abstract}
We prove a new two-cardinal theorem, e.g. $\left(\boldsymbol{\aleph}_{\omega}, \boldsymbol{\aleph}_{0}\right) \rightarrow\left(2^{\boldsymbol{K}_{0}}, \boldsymbol{\aleph}_{0}\right)$.
\end{abstract}
For this we prove a combinatorial theorem.

This is a sequel of Shelah [S1], and solves the main problem there. This problem also appears in Chang and Keisler $[\mathbf{C K}]$ and Friedman $[\mathbf{F r}$, Problem 30]. Our result is:

THEOREM 1. (A) If for every $n<\omega$, the first order theory $T$ has a model type $\left(\boldsymbol{\aleph}_{\alpha+n}, \boldsymbol{\aleph}_{\alpha}\right)$ then whenever $|T| \leqslant \mu<\lambda<\operatorname{Ded}^{*} \mu$, T has a model type $(\lambda, \mu)$.

(B) If $\boldsymbol{\aleph}_{\alpha+\omega}<\operatorname{Ded}^{*} \boldsymbol{\aleph}_{\alpha}$ then $\left(\boldsymbol{\aleph}_{\alpha+\omega}, \boldsymbol{\aleph}_{\alpha}\right)$ is $\boldsymbol{\aleph}_{\alpha}$-compact and is complete.

REMARK. Ded ${ }^{*} \mu$ is the first cardinal $\chi$ such that no tree with $\leqslant \mu$ nodes has $\geqslant \chi$ branches of the same height. Note that $\operatorname{Ded}^{*} \aleph_{0}=\left(2^{\aleph_{0}}\right)^{+}$, for every $\lambda \lambda^{+}<\operatorname{Ded}^{*} \lambda \leqslant\left(2^{\lambda}\right)^{+}$, and it is consistent with ZFC that $\operatorname{Ded}^{*} \boldsymbol{\aleph}_{1} \leqslant 2^{\boldsymbol{N}_{1}}$.

This leads to many conjectures whose difficulty is not known to me; a sample is:

ConjeCture 2. (A) $\left(\aleph_{\alpha+\omega+\omega}, \aleph_{\alpha+\omega}, \aleph_{\alpha}\right) \rightarrow(\lambda, \mu, \chi)$ whenever $\chi<\mu<\lambda$ $<\operatorname{Ded}^{*} \chi$.

(B) If a countable theory $T$ has a $\lambda$-like model, $\lambda$ a limit cardinal, and $|T| \leqslant \mu<\lambda_{1}<\operatorname{Ded}^{*} \mu, \lambda_{1}$ a singular cardinal then $T$ has a $\lambda_{1}$-like model. If $\lambda$ is $M_{\omega}$-Mahlo weakly inaccessible cardinal, we can remove the singularity of $\lambda_{1}$.

(C) If $\psi \in L_{\omega_{1}, \omega}$ has a model of cardinality $\aleph_{\omega_{1}}$, then $\psi$ has a model of cardinality $2^{\aleph_{0}}$.

Notation. Let $I$ denote a well-ordered set. A $(\lambda, n)$-box $B$ is $\Pi_{l<n} I_{l}$ where $I_{l}$ has order type $\lambda ; \lambda, \mu, \chi$ denote infinite cardinals, elements of boxes will be denoted by $\eta, \tau, v$, and $\eta=\langle\eta(0), \ldots, \eta(n-1)\rangle$. For a $(\lambda, n)$-box $B$, and $\eta_{l} \in B(l<n)$ we say $\left\langle\eta_{0}, \ldots, \eta_{n-1}\right\rangle$ is proper for $B$ if $k \neq l<n \Rightarrow \eta_{k}(l)$ $<\eta_{l}(l)$.

Let $\lambda^{+0}=\lambda, \lambda^{+(k+1)}=\left(\lambda^{+k}\right)^{+}=$the successor of $\lambda^{+k}$.

A $B$-indexed set is $\left\{a_{\eta}: \eta \in B\right\}$ such that $\eta \neq \tau \rightarrow a_{\eta} \neq a_{\tau}$. Under those conditions $\left\langle a_{\eta_{0}}, \ldots\right\rangle$ is proper, iff $\left\langle\eta_{0}, \ldots\right\rangle$ is proper. $\mathrm{A}(\lambda, n)$-indexed set is a $B$-indexed set for some $(\lambda, n)$-box $B$.

LEMMA 3. Suppose that $f_{\alpha}:\left(\lambda^{+}\right)^{2} \rightarrow \lambda$ for each $\alpha<\lambda$. Then there exist

Received by the editors November 27, 1974 and, in revised form, May 1, 1975.

AMS (MOS) subject classifications (1970). Primary 02H05, 04A20.

Key words and phrases. Two-cardinal theorem, partition calculus.

${ }^{1}$ I would like to thank the referee for detecting many errors, and rewriting Lemma 3 -Theorem 5. 
$s, t<\lambda^{+}$such that for each $\alpha<\lambda$ there exist $a, b, c, d$ for which $s<a<b$ $<\lambda^{+}, t<c<d<\lambda^{+}$and $f_{\alpha}(s, t)=f_{\alpha}(a, d)=f_{\alpha}(b, c)$.

Proof. For every $u<\lambda^{+}$let $t_{u}<\lambda^{+}$be such that whenever $\alpha<\lambda$ and $t \geqslant t_{u}$, then

$$
\left|\left\{v<\lambda^{+}: f_{\alpha}(u, t)=f_{\alpha}(u, v)\right\}\right|=\lambda^{+} .
$$

Let $X_{u}=\left\{(\alpha, \beta): f_{\alpha}(u, t)=\beta\right.$ for some $\left.t \geqslant t_{u}\right\}$. Now let $s<\lambda^{+}$be such that whenever $u \geqslant s$ and $(\alpha, \beta) \in X_{u}$, then

$$
\left|\left\{v<\lambda^{+}:(\alpha, \beta) \in X_{v}\right\}\right|=\lambda^{+} \text {. }
$$

Let $t=t_{s}$. It is clear that this $s$ and $t$ work.

LEMma 4. Let $f: A^{n} \rightarrow \lambda$ where $A$ is any $\left(\lambda^{+}, n+1\right)$-indexed set and $k<n$. Then there is a $(\lambda, n)$-indexed set $A^{*} \subset A$ such that:

(*) For any proper sequence $\left\langle a_{0}, \ldots, a_{n-1}\right\rangle$ from $A^{*}$ there is a proper sequence $\left\langle b_{0}, \ldots, b_{n}\right\rangle$ from $A$ such that

$$
f\left(a_{0}, \ldots, a_{n-1}\right)=f\left(b_{0}, \ldots, b_{n-1}\right)=f\left(b_{0}, \ldots, b_{k-1}, b_{n}, b_{k+1}, \ldots, b_{n-1}\right) .
$$

Proof. Let $A$ be a $B$-indexed set where $B=\prod_{l<n+1} I_{l}$ and $A=\left\{a_{\eta}: \eta\right.$ $\in B\}$. For notational simplicity let $k=n-1$ and each $I_{l}=\lambda^{+}$. Now we define $\left\langle s_{\alpha}: \alpha<\lambda\right\rangle$ and $\left\langle t_{\alpha}: \alpha<\lambda\right\rangle$ by induction on $\alpha$ such that:

(i) $s_{\alpha}, t_{\alpha}<\lambda^{+}$.

(ii) $\left\langle s_{\alpha}: \alpha<\lambda\right\rangle$ and $\left\langle t_{\alpha}: \alpha<\lambda\right\rangle$ are increasing.

(iii) Whenever $\eta_{0}, \ldots, \eta_{n-2} \in B$ and $\tau \in \lambda^{n-1}$ are such that for each $i, l<n-1$ there is $\beta<\alpha$ such that $\eta_{i}(l)<\lambda, \eta_{i}(n-1)=s_{\beta}$ and $\eta_{i}(n)$ $=t_{\beta}$, then there are $a, b, c, d$ such that $s_{\alpha}<a<b<\lambda^{+}, t_{\alpha}<c<d<\lambda^{+}$ and

$$
\begin{aligned}
f\left(a_{\eta_{0}}, \ldots, a_{\eta_{n-2}}, a_{\tau \wedge\left\langle s_{\alpha}, t_{\alpha}\right\rangle}\right) & =f\left(a_{\eta_{0}}, \ldots, a_{\eta_{n-2}}, a_{\tau \wedge\langle a, d\rangle}\right) \\
& =f\left(a_{\eta_{0}}, \ldots, a_{\eta_{n-2}}, a_{\tau \wedge\langle b, c\rangle}\right) .
\end{aligned}
$$

Suppose we have defined $s_{\beta}$ and $t_{\beta}$ for $\beta<\alpha$. For each $\eta_{0}, \ldots, \eta_{n-2}, \tau$ satisfying the conditions of (iii), there is a function $g:\left(\lambda^{+}\right)^{2} \rightarrow \lambda$ defined by

$$
g(x, y)=f\left(a_{\eta_{0}}, \ldots, a_{\eta_{n-2}}, a_{\tau \wedge\langle x, y\rangle}\right) .
$$

There are $\leqslant \lambda$ such functions $g$. So we can apply Lemma 3 to get $s_{\alpha}, t_{\alpha}$ such that $s_{\alpha}>t_{\beta}$ and $t_{\alpha}>t_{\beta}$ for each $\beta<\alpha$, and for each such $g$ there are $a, b, c, d$ such that $s_{\alpha}<a<b<\lambda^{+}, t_{\alpha}<c<d<\lambda^{+}$and $g\left(s_{\alpha}, t_{\alpha}\right)=g(a$, d) $=g(b, c)$.

Now we define the $(\lambda, n)$-indexed set $A^{*}$. For each $\tau \in \lambda^{n-1}$ let $b_{\tau \wedge}\langle\alpha\rangle$ $=a_{\tau \sim\left\langle s_{\alpha}, t_{\alpha}\right\rangle}$. Then let $A^{*}=\left\{b_{\eta}: \eta \in \lambda^{n}\right\}$. Now it is easy to check that (*) holds.

THEOREM 5. Let $f_{l}:\left(\lambda^{+n}\right)^{l} \rightarrow \lambda$ whenever $0<l \leqslant n$, and let $h:(n+1) \rightarrow n$ be such that $h(l)<l$ whenever $0<l \leqslant n$. Then there are distinct $a_{0}, \ldots, a_{n}$ $<\lambda^{+n}$ such that 


$$
f_{l}\left(a_{0}, \ldots, a_{l-1}\right)=f_{l}\left(a_{0}, \ldots, a_{h(l)-1}, a_{l}, a_{h(l)+1}, \ldots, a_{l-1}\right)
$$

whenever $0<l \leqslant n$.

Proof. We let $\lambda^{+n}$ be $\left(\lambda^{+n}, n+1\right)$-indexed. Now we prove the theorem by induction on $n$. For $n=0$ there is nothing to prove. For $n+1$ we use Lemma 4, and the induction hypothesis on $A^{*}$.

Proof of Theorem 1. Clear from [S1, §3] and Lemma 4.

REMARKS. (1) The following theorem is clear.

THEOREM . If every finite subset of $T$ has, for each $n$, a model of type $\left(\lambda_{m}, \ldots, \lambda_{0}\right)$ where $\lambda_{0}^{+n}<\lambda_{1},\left[\left(\lambda_{l+1}\right)^{\left(\lambda_{l}^{+n}\right)}\right]^{+n}<\lambda_{l+2}(l<n-1)$ and $|T| \leqslant \mu_{0}$ $\leqslant \mu_{1} \leqslant \cdots \leqslant \mu_{m}<\operatorname{Ded}^{*} \mu_{0}$ then $T$ has a model of type $\left(\mu_{m}, \ldots, \mu_{0}\right)$. (The parallel theorem in [S1] was noted by Papageorgiou.)

(2) We can prove the main theorem of [S1] in a way similar to the proof here.

(3) In the notation of Shelah [S2, §3], we have proved in Lemma 4 that for $m=2^{n}, r<\omega, \lambda^{+m} \stackrel{w t}{\longrightarrow}(n)_{\lambda}^{r}$. This answers positively question 3 from [S2]. But it is still unknown whether we have the best results.

(4) Halperin and Levi [H Le] used an indiscernibility similar to the one used in [S1], and Halperin and Lauchli proved the necessary combinatorial theorem. We have not succeeded in generalizing their proof. However, we can use our method to prove a weaker variant of their theorem, which is sufficient to prove that if $T \subset T_{1},\left|T_{1}\right|=\aleph_{0}, T$ is complete and there are $>\aleph_{0}$ complete $L(T)$-types consistent with $T, \lambda>\aleph_{0}$ then $T$ has $\geqslant \min \left\{2^{\lambda}, 2^{2^{\kappa_{0}}}\right\}$ nonisomorphic $L(T)$-reducts of models of $T_{1}$. (This will appear in [S3].)

(5) To see the connection note that by [S3] it follows by Theorem 5 that

THEOREM . Let $f_{l}:\left(\lambda^{+n}\right)^{l} \rightarrow \lambda$ whenever $0<l \leqslant n, n=2^{m}-1$. Then there are distinct $a_{\eta}<\lambda^{+n}$ for $\eta \in 2^{m}$ (i.e. $\eta$ is a sequence of ones and zeros of length $m$ ) such that: if $k<m, 0<l \leqslant n, \tau_{1}, \ldots, \tau_{l}$ are distinct members of $2^{k}$, and for $1 \leqslant i \leqslant l, 0 \leqslant j \leqslant 1, \eta_{i}^{j} \in 2^{m}$ and $\tau_{i}$ is an initial segment of $\eta_{i}^{j}$ then $f_{l}\left(\eta_{1}^{0}, \ldots, \eta_{l}^{0}\right)=f_{l}\left(\eta_{1}^{1}, \ldots, \eta_{l}^{1}\right)$.

(6) In Lemma 4 and Theorem 5 we can consider $\mu$ such functions, provided that $\mu \leqslant x, \lambda^{\mu}=\lambda$, resp.

(7) We can use only a $(B, n)$-indexed set for a fixed $n$ in (4), but then in Remark (3) $m$ will become bigger.

\section{REFERENCES}

[CK] C. C. Chang and H. J. Keisler, Model theory, North-Holland, Amsterdam, 1973.

[H Le] J. D. Halpern and A. Lévy, The Boolean prime ideal theorem does not apply the axiom of choice, Proc. Sympos. Pure Math., vol. 13, part 1, Amer. Math. Soc., Providence, R.I., 1971, pp. 83-134. MR 44 \# 1557.

[H La] J. D. Halpern and H. Lauchli, A partition theorem, Trans. Amer. Math. Soc. 124 1966, 360-367. MR 34 \#71.

[S1] S. Shelah, A two-cardinal theorem, Proc. Amer. Math. Soc. 48 (1975), 207-213.

[S2] - Coloring without triangles and partition relation, Israel Math. 20 (1975), 1-12.

[S3] - Stability and number of non-isomorphic models (in preparation).

Institute of Mathematics, The Hebrew University, Jerusalem, Israel 